\title{
Playing with Tactile Feedback Latency in Touchscreen Interaction: Two Approaches
}

\author{
Topi Kaaresoja ${ }^{1}$, Eve Hoggan ${ }^{2}$, and Emilia Anttila ${ }^{3}$ \\ ${ }^{1}$ Nokia Research Center, Helsinki, Finland \\ ${ }^{2}$ Helsinki Institute of Information Technology HIIT, University of Helsinki, Finland \\ ${ }^{3}$ Nokia, Espoo, Finland \\ topi.kaaresoja@nokia.com, eve.hoggan@helsinki.fi, \\ emilia.anttila@nokia.com
}

\begin{abstract}
A great deal of research has investigated the potential parameters of tactile feedback for virtual buttons. However, these studies do not take the possible effects of feedback latencies into account. Therefore, this research investigates the impact of tactile feedback delays on touchscreen keyboard usage. The first experiment investigated four tactile feedback delay conditions during a number entry task. The results showed that keypads with a constant delay $(18 \mathrm{~ms})$ and the smallest feedback delay variation were faster to use and produced less errors compared to conditions with wider delay variability. The experiment also produced an unexpected finding - users seemed to perceive buttons with longer delays as heavier, with a need for greater force when pressing. Therefore another experiment was conducted to investigate this phenomenon. Seven delay conditions were tested using a magnitude estimation method. The results indicate that using different latencies can be used to represent tactile weight in touchscreen interaction.
\end{abstract}

Keywords: Touchscreen, latency, tactile feedback, weight.

\section{Introduction}

Nowadays, many devices in different product domains support multimodal interaction. For example, touchscreen mobile phones now provide not only visual feedback but also auditory and haptic responses to user input. Despite the fact the microprocessors are becoming increasingly fast, operating systems and applications are more complex thus placing higher demands on the processors. This can lead to latency and latency variation in interaction. The research described in this paper focuses on different aspects of tactile latency in touchscreen interaction.

In the area of HCI research, latency is traditionally viewed as being harmful to interaction due to its potentially disruptive effect on usability and the user experience [1-3]. However, previous psychophysical latency research indicates that stimuli from different modalities must appear asynchronously to be perceived as simultaneous [46]. Therefore, in some cases, latency must be intentionally introduced into feedback. Our previous work introduce these latency issues more in detail [7, 8]. 
This paper will address the following questions: How do constant and variable tactile feedback latencies affect performance and perceived pleasantness? How does audio feedback inherently part of the tactile feedback affect the perception of tactile feedback latencies? It must also be noted that certain perceptual consequences, such as illusions, may occur when latency is introduced to tactile feedback. Therefore, our final research question asks whether illusions created through latency can be used as a design parameter for an interface when carefully selected and controlled.

This paper reports the results of two experiments focusing on tactile latency. The first experiment investigated four different tactile feedback delay variation conditions during a number entry task on a touchscreen keypad. The second experiment was conducted to test seven different delay conditions for virtual buttons using a magnitude estimation method. This experiment was used to determine whether using different latencies can be an effective way in which to represent tactile weight as a parameter in touchscreen interaction.

\section{Background Work}

The following section will briefly describe some of the existing research in the areas of tactile feedback for touchscreens and latency tolerance in relation to this work.

\subsection{Touchscreens with Tactile Feedback}

Recent research has mainly focused on the technology used to provide the tactile feedback from touchscreens on mobile devices and also on the advantages of augmenting visual displays with an additional modality through which information can be transmitted.

Poupyrev et al. [9] introduce tactile feedback for ,mobile touchscreen devices. They embedded TouchEngine, a thin, miniature low-power tactile actuator in a PDA. The actuator was designed specifically for use in mobile interfaces, and it is a piezoelectric actuator that bends when a signal is applied. In this case, Poupyrev et al. used the tactile feedback as an ambient background channel of information for several applications. They conducted a formal user study into the use of tactile feedback with tilting devices, for example. Participants were required to scroll through a text list using gestures. The results of the study showed that, on average, participants could complete the tasks $22 \%$ faster when provided with tactile feedback.

Nashel and Razzaque [10] added tactile cues simulating real buttons to virtual buttons displayed on mobile devices with touch screens. As the user's finger moves over a virtual button: a 'pop' is presented as the finger enters a button region; a low amplitude vibration is presented when the finger pushes the button; a short pulse is presented as the finger leaves the button area and no feedback when the finger is between buttons. The experiments conducted found that all participants were able to differentiate between vibration (finger over a button) and no vibration (finger not over any button).

Kaaresoja et al. [11] presented a touchscreen mobile device augmented with piezoelectric tactile feedback. The actuators are positioned under a resistive touchscreen, and can provide tactile feedback to a stylus or finger. The authors suggest four applications for the touchscreen tactile feedback: numerical keypad, text selection, scrolling, and drag 
and drop. Lastly, Hoggan et al. [12] presented a study investigating the use of tactile feedback for touchscreen keyboards on PDAs. The tactile feedback added to the standard touchscreen buttons was made up of simple tactons, that are abstract structured vibrotactile icons that can be used to encode multiple dimensions of information [13].

The research mentioned above shows that tactile feedback can be an effective addition to touchscreen interaction in terms of performance and user satisfaction. However, even though most tactile hardware involves at least a small amount of latency, the majority of this research does not take this into account. The promising results of the studies discussed above may be drastically reduced if users are not tolerant to latencies.

\subsection{Latency}

Adelstein et al. [14] experimented with the threshold for detecting a difference between two different latency conditions between audio and tactile stimuli. The participants tapped a brick with the rubber tip of a hammer twice, and were asked to judge if there was difference in the lag of two stimuli pairs. The results suggest that the just noticeable difference is $24 \mathrm{~ms}$, which as unaffected by the length of the audio stimulus.

Mäki-Patola and Hämäläinen [15] studied the tolerance of a human performer for latency in gesture controlled musical instruments. The threshold for latency perception was between 20 and $30 \mathrm{~ms}$. They also found out that the playing style affected the detection of latency: if slow passages of music with vibrato were played, high latencies were tolerated. Also younger participants detected the latency more easily.

MacKenzie and Ware [16] reported a study on the effect of latency in a target acquisition task using the Fitts' law paradigm. They introduced four latency conditions, $8.3,25,75$ and $225 \mathrm{~ms}$. The task was to move a small rectangle to a target with a mouse on a computer screen. They modified the target width and the distance from the target. The results showed that the maximum latency $225 \mathrm{~ms}$ significantly slowed down the task by $64 \%$ from the reference $8.3 \mathrm{~ms}$, error rate being $214 \%$ higher (3.6\% to $11.6 \%$ ).

In our previous work [8] we investigated the effect of constant tactile feedback latency in touchscreen keypad interaction. We experimented with latency between the virtual button press and the tactile feedback from $18 \mathrm{~ms}$ to $118 \mathrm{~ms}$.. The task was to enter numbers in a number keypad and short sentences in a QWERTY keypad. The latency was unchanged during a test block of number or character entry tasks. The results showed that the users tolerate the tactile feedback latency well. The performance did not drop significantly. However, an effect was found in subjective satisfaction: the satisfaction drops as the delay gets longer.

Although a small amount of research has been conducted in the area of tactile feedback latency for touchscreens, there has been no investigation of variable latencies or of the potential design opportunities provided by such latencies.

\section{Experiment 1: Variable Delay}

The first experiment in this research investigated the effect of tactile feedback latency variation on user performance in touchscreen interaction. The aim of the study was to compare the efficiency, accuracy and subjective perceived pleasantness of the touchscreen use between different tactile feedback latency variation conditions. 


\subsection{Participants}

A within subjects design was used with 12 participants. Six of them were female and six were male. The age varied from 18 years to 47 , average age being 28 years. All the participants were employees of Nokia Research Center. They were all right-handed. Ten of the participants had used a mobile phone for over five years and two of them for about 3-5 years. All participants claimed they write an average of three text messages per day. Four participants said they use a touchscreen device daily. Five participants said that they use touchscreen devices once in a while, and the last three participants had never used a touchscreen device.

\subsection{Equipment and Stimuli}

Experiment 1 was conducted on a Nokia 770 Internet Tablet (Fig. 1) augmented with tactile feedback [17]. The 4-inch $(90 \times 54 \mathrm{~mm})$ touchscreen displayed a virtual numeric keypad (Fig. 2). The experiment application was written in Python. Tactile feedback was generated with two piezo discs which were placed under the display module [17].

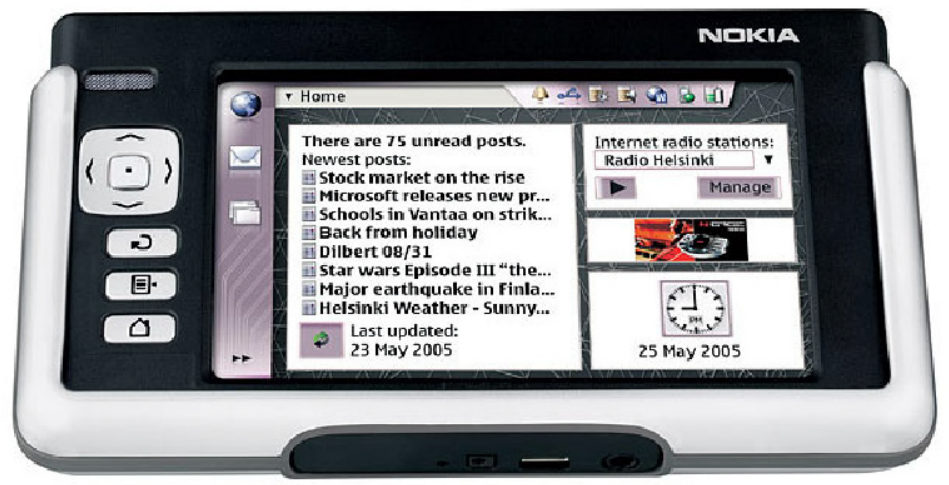

Fig. 1. Nokia 770 Internet Tablet enhanced with tactile feedback

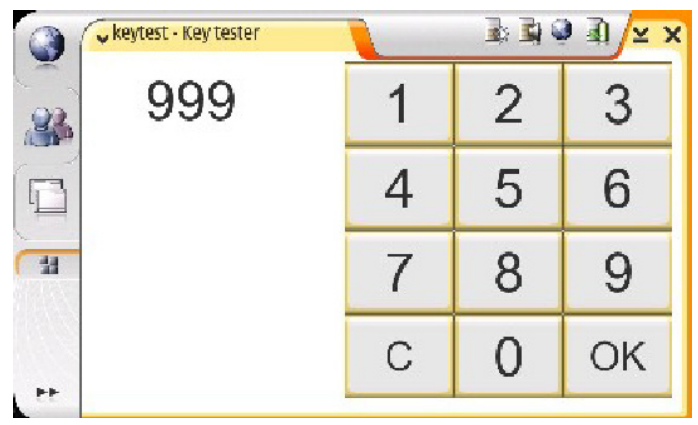

Fig. 2. The touchscreen displayed a virtual keypad 
We decided to study button-down feedback as opposed to button-down and buttonup to keep the number of experiment conditions low enough and because an experiment with button-up feedback was conducted separately [8]. Button-up feedback on touchscreens can be complicated because the user's finger tends to have been removed from the display before the feedback is presented meaning that tactile feedback can often go unnoticed at least when the latency is higher.

The tactile pulse used in the Experiment 1 is shown in Fig. 3. The rise time of the pulse was $1 \mathrm{~ms}$ and the fall time $4 \mathrm{~ms}$, displacement of the display module being about $6 \mu \mathrm{m}$. When the user pressed a key, this pulse was given after a delay described below.

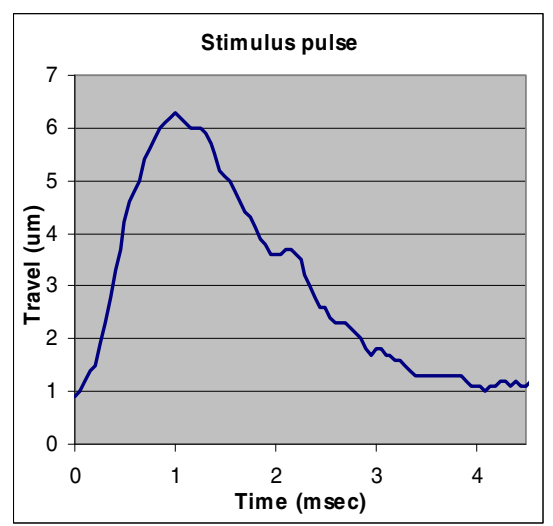

Fig. 3. The tactile feedback pulse used in Experiment 1. The figure shows the displacement of the display module.

Four different tactile feedback latency variation conditions were selected. The first condition was the reference $18 \mathrm{~ms}$ (minimum latency achieved with the hardware used) with no variation. The second condition was latency variation between 18 and $36 \mathrm{~ms}$ (randomized equal amount of latencies 18 and $36 \mathrm{~ms}$ ), the third being between 18 and 72 (randomized equal amount of latencies 18, 36 and $72 \mathrm{~ms}$ ), and the fourth between 18 and $144 \mathrm{~ms}$ (randomized equal amount of latencies 18, 36, 72 and 144). As we can see, the maximum latency was doubled for every condition. The latency variation conditions were named as 18, 18-36, 18-72 and 18-144. The delay was validated with a microphone and an oscilloscope.

\subsection{Procedure}

The piezo technology inherently produces audio feedback in addition to tactile feedback. In order to find out if the audio feedback has an effect, we decided to run the experiment both with and without headphones. In the first condition, typical street noise was played in the headphones to mask the audio feedback from the device. These two environmental conditions were named as Tactile (with headphones) and Tactile\&Audio.

The task was to key in the three numbers, which appeared on the display at once and press lastly the OK button using the virtual keypad (Fig. 2). If the participants 
made an error, they could correct it with the C-button. There were altogether 55 tasks in one block. The block was repeated in all four conditions. There were 440 tasks in Experiment (55 tasks, 4 latency conditions and 2 environmental conditions) altogether. All the blocks and conditions were counterbalanced. The experiment application measured the time from the first keypress to the keypress of OK button and it also wrote the numbers, the test user keyed in, into a results file.

Before starting the actual experiment, the participants were able to try to key in few three digit number series. The participants were instructed to hold the device with both hands and press the virtual buttons on the touchscreen with their right thumb (Fig. 4).

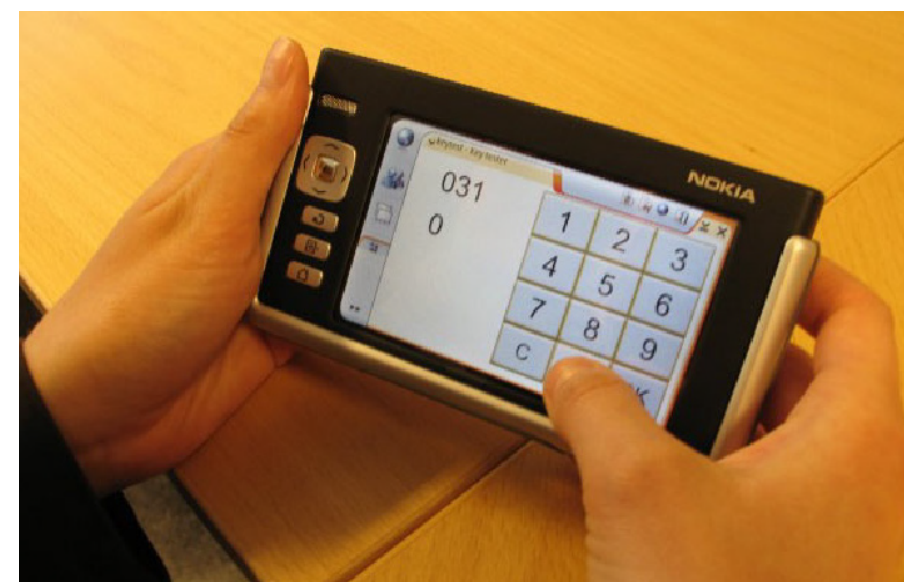

Fig. 4. The participants were instructed to hold the device with their both hands and press the virtual buttons with their right hand's thumb

There was a questionnaire concerning the subjective satisfaction after each block. The participants were asked to rate their degree of agreement with the following statements using a 1-7 rating scale, where 1 meant totally disagree and 7 totally agree:

- This keypad is pleasant to use.

- I felt myself comfortable when using this keypad.

- Pressing the keypad buttons felt just like pressing physical ("real") buttons.

- I always knew that the device received my keypress.

- I would like to buy a device with this kind of keypad.

There also was a interview containing the following questions after the completion of all the test blocks:

1. Did you notice any difference in the feedback?

2. Did you notice that some of the feedback came after a delay?

3. What did you think about the feedback delay? Was it distracting? Did the feedback delay have some effect on your performance?

It took approximately 30 minutes to complete the whole experiment. 


\subsection{Results}

Fig. 5 shows the average task time with $95 \% \mathrm{CI}$ in all the latency variation conditions for both Tactile and Tactile\&Audio conditions. A two-way repeated measures ANOVA showed there is a significant difference in the latency variation condition $[\mathrm{F}(3,33)=2.72, \mathrm{p}=0.049]$. Post-hoc Tukey HSD analysis showed the statistical difference are between the [Tactile\&Audio 18] and [Tactile\&Audio 18-144] $(\mathrm{p}<0.05)$, [Tactile\&Audio 18-36] and [Tactile\&Audio 18-144] $(\mathrm{p}<0.05)$, [Tactile\&Audio 18] and [Tactile 18-144] $(\mathrm{p}<0.05)$, and [Tactile\&Audio 18-36] and [Tactile 18-144] $(\mathrm{p}<0.05)$. It means that the participants performed significantly faster with the reference $(18 \mathrm{~ms})$ and the minimum latency variation (18-36 ms) without headphones than with the largest latency variation (18-144 ms) with or without headphones. The analysis did not show statistical difference between the Tactile and Tactile\&Audio conditions $[\mathrm{F}(1,11)=0.51, \mathrm{p}=0.51]$.

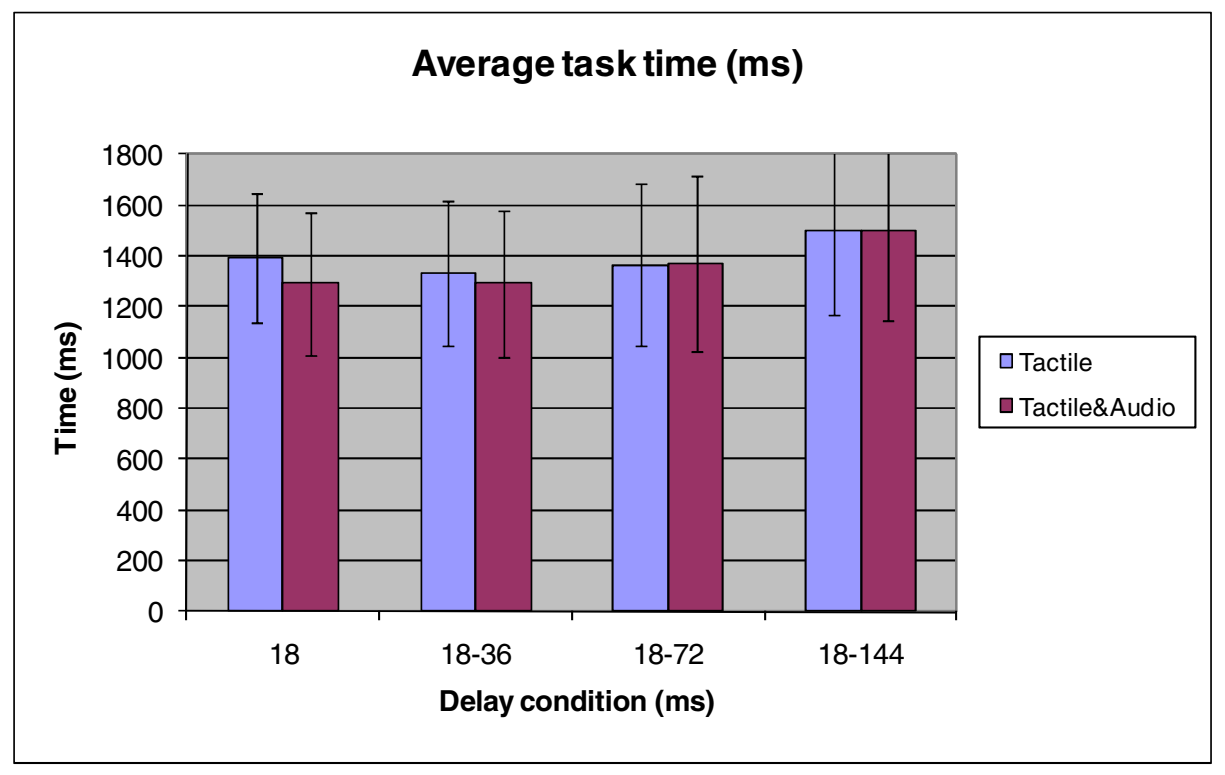

Fig. 5. The average task time with $95 \% \mathrm{CI}$ in with and without headphones

Fig. 6 shows the average error rate with 95\%CI in all the latency variation conditions for both Tactile and Tactile\&Audio conditions. A two-way repeated measures ANOVA showed there is no significant difference in the latency variation condition $[\mathrm{F}(3,33)=2.43, \mathrm{p}=0.07]$, or the between Tactile and Tactile\&Audio conditions $[\mathrm{F}(1,11)=1.39, \mathrm{p}=0.24]$. 


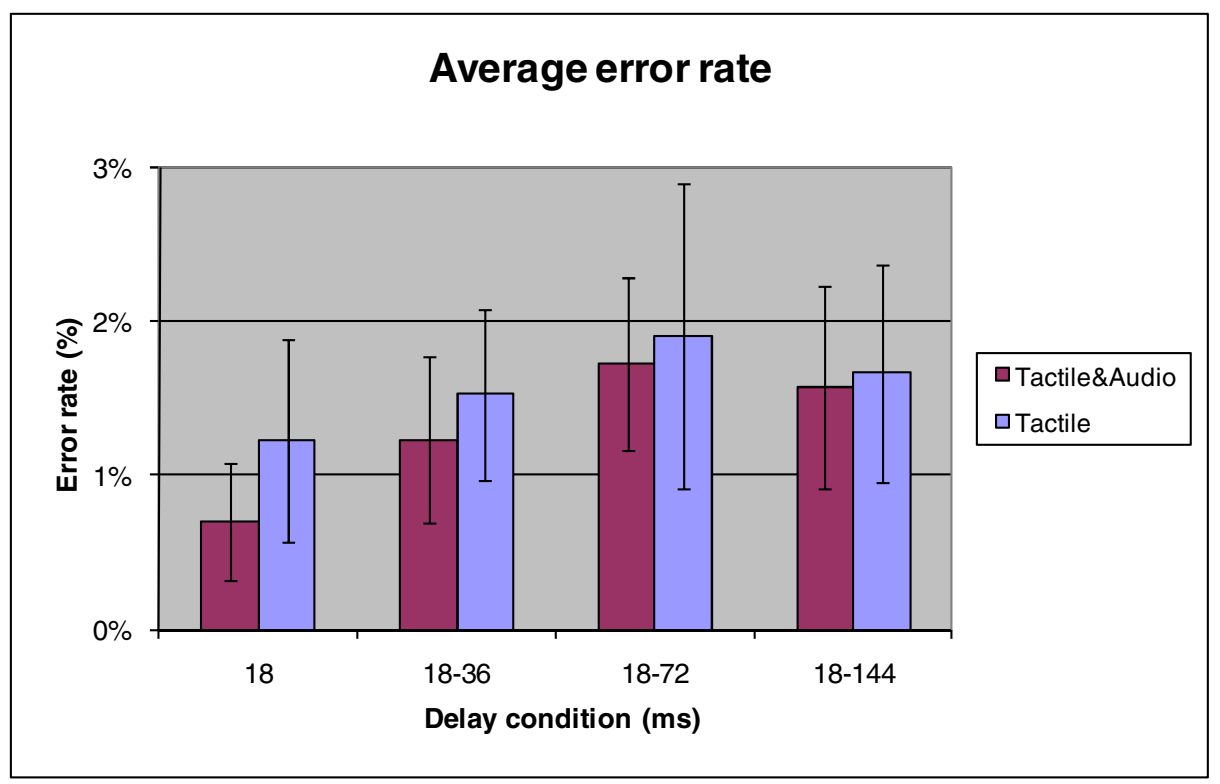

Fig. 6. The average error rate with $95 \% \mathrm{CI}$ in with and without headphones

Fig. 7 and Fig. 8 show the mean values and standard deviations for degree of agreement with the satisfaction questionnaire statements on a 1 to 7 (totally disagreetotally agree) scale in Tactile and Tactile\&Audio conditions. In both conditions the results show that the keypad with largest delay variation was clearly rated lowest in all five statements. Therefore we can conclude that it is less pleasant to use compared to other keypads with less delay variation.

In the interview, the users commented that, without the audio feedback (with the headphones on), the significance of the tactile feedback was quite obvious. They noticed differences in the keypad's sensitivity and accuracy. Participants stated that keypad with the largest delay variation required more force to press the buttons and felt somewhat unreliable. Only a couple of users noticed the delay in some of the feedback and the others did not notice that at all (10 out of 12 participants). The keypad with the shortest delay was said to be the most pleasant to use; it was fast and felt natural.

Users without the headphones (Tactile\&Audio condition) commented that they noticed differences in the keypad's accuracy and reliability. The keypad with the shortest delay was said to be more sensitive and reliable and it did not require so much force to press the buttons compared to other keypads. Only two of the test users said that they noticed that some feedback came after a delay but the rest did not notice that at all. However, almost all users commented that there was something seriously wrong with the keypad with the largest delay variation and it was horrible to use but they could not figure out the reason behind this. Users commented that they needed to press the buttons very hard before the keypad received their key-press. 


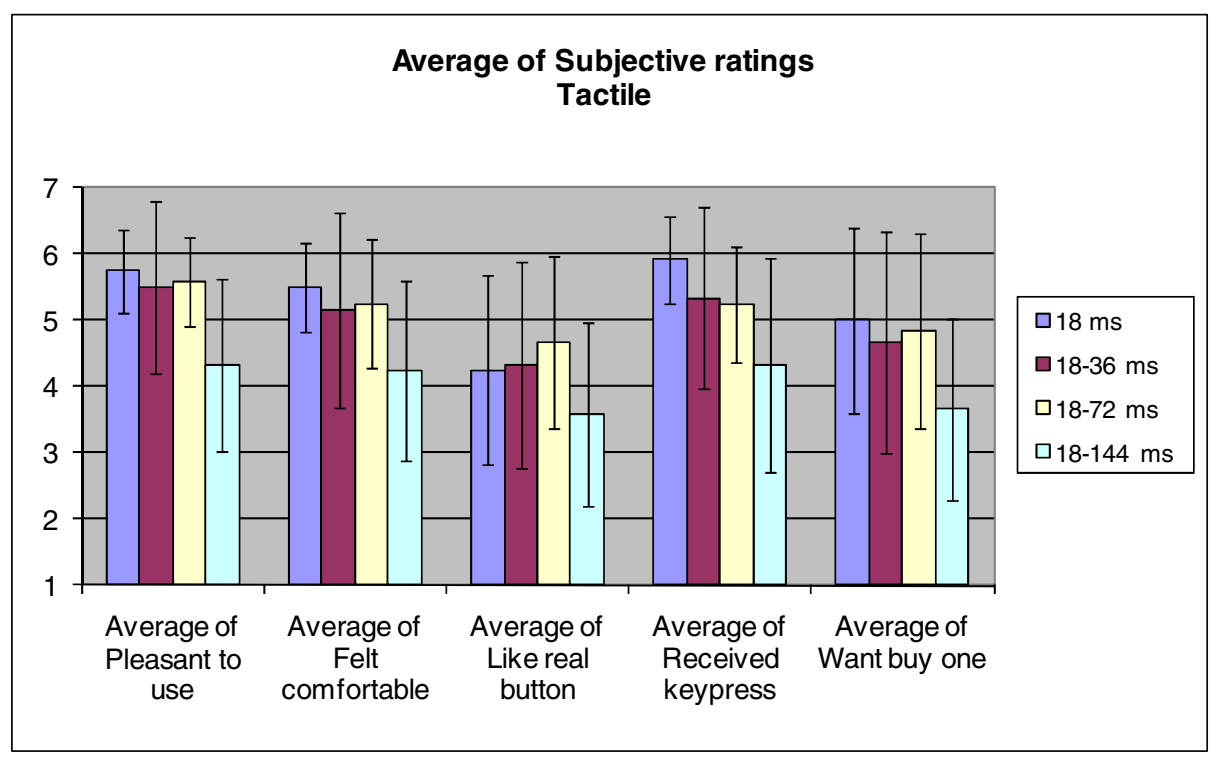

Fig. 7. Mean values and standard deviations of subjective ratings in Tactile condition

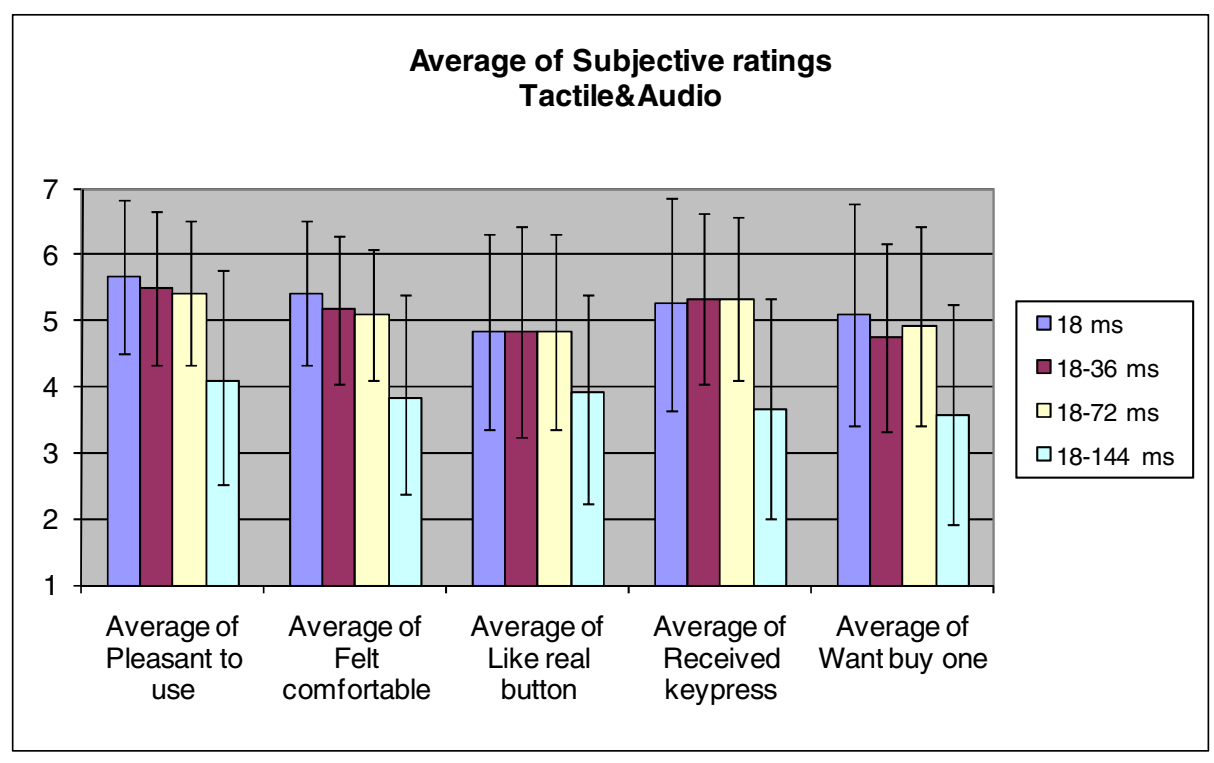

Fig. 8. Mean values and standard deviations of subjective ratings in Tactile\&Audio condition

\subsection{Discussion}

This study clarified the effect of tactile feedback delay variation on the user performance in the use of the touchscreen device. The study compared the efficiency, 
accuracy and subjective perceived pleasantness of touchscreen use between different tactile feedback delay variation conditions. The experiment also investigated whether audio feedback biases the effect of tactile feedback delay variation on user performance and the evaluation of the pleasantness of the tactile feedback.

It was found that the keypad with a feedback delay variation of 18-144 ms was slowest to use. However, there was no significant increase in the error rate. The results also showed that in the satisfaction questionnaire the keypad with the largest delay variation 18-144 ms received clearly the lowest score in all five statements compared to other delay conditions.

Therefore it is suggested that users can tolerate a tactile feedback delay varying from $0 \mathrm{~ms}$ to $36 \mathrm{~ms}$ and even $76 \mathrm{~ms}$ when using touchscreen buttons. However, wider feedback delay variations impact user performance, with reduced accuracy and increased task times, and are thereby unacceptable. The same conclusion can be drawn from the pleasantness evaluations where users strongly disliked the keypad with the biggest delay variation.

The pairwise comparisons between the Tactile\&Audio and Tactile conditions showed no statistical differences. These results indicate that inherent audio feedback does not have an effect on user performance or the perceived pleasantness of touchscreen buttons incorporating tactile feedback variations.

\section{From Performance Measurements to Illusions}

The experiment described next takes the issue of tactile latency one step further by examining the perceptual illusions. In the areas of HCI and UI design, delays in feedback for a user's actions are often considered as negative issues and are to be avoided at all costs. However, the results of the experiment detailed above have shown that latency in tactile feedback is varying between $0 \mathrm{~ms}$ to $36 \mathrm{~ms}$ in touchscreen virtual button use cases is acceptable. In fact, in Experiment 1, most users (10 out of 12) were not aware of the delay. Interestingly when participants were asked in post-study questionnaires about their experience during the experiments, several participants mentioned aspects related to the size and weight of the buttons. For example, in Experiment 1, one participant stated, "especially the last one (18-144 ms) required much more force to press the buttons" and "I needed to press the buttons very hard". A previous study [8] on constant feedback latencies produced similar findings where participants felt that they needed to apply more force to the touchscreen buttons when the delay was longer, even though the activation threshold of the touchscreen was not altered in any way. It appears as though the introduction of different delays to tactile feedback does not affect latency perception but in fact affects the perception of the size/weight of the button.

\section{Experiment 2: Perceived Weight Study}

Finding design parameters for tactile feedback to encode information is a difficult task as the set of parameters that can be manipulated is much smaller than in sound 
for example, and this reduces the number of stimuli that can be created. Researchers are trying to find effective new parameters that can be used for the design of distinguishable tactile feedback. Previous studies have investigated parameters such as rhythm, spatial location and texture [13, 18, 19]. The post-study questionnaire results in Experiment 1 suggest that latency may be a potential new parameter for use in the design of tactile feedback. Introducing latency may be sufficient to create a full-strength illusion of weight or depth.

In an effort to establish weight (created using latency) as a tactile feedback design parameter, this section presents the results from an experiment investigating whether users perceive buttons with a bigger delay as being heavier or larger. We present practical implementation details and guidelines based on the results focusing on the design of heavy/light touchscreen buttons and more specifically, on the most effective parameter design. Drawing on the results, we argue that weight can be used as an additional parameter when designing tactile feedback for touchscreens.

\subsection{Related Work}

Traditionally most research into the perception of weight has concluded that the visual sense has dominance over the other senses. In particular, the most famous area of study is the size weight illusion [20-22] where when lifting two objects of different volume but equal weight, people judge the smaller object to be heavier.

However, more recently it has been shown that the sense of touch can also affect the perception of weight. Ellis and Lederman [23] showed that the size-weight illusion is a primarily haptic phenomenon where haptic volume cues can create the same size-weight illusion as well as visual volume cues if not better. Amazeen [24] also showed that a dependence of perceived heaviness relies on a dependence on perceived volume (perceptual independence). This volume can be perceived through the sense of touch by allowing users to grasp virtual objects using force feedback devices for example.

These results would suggest that it might be beneficial to investigate the use of haptic volume cues in touchscreen interaction too. Although the objects cannot be physically grasped, it may be possible to create a similar illusion of weight or volume without using visual cues.

\subsection{Equipment and Stimuli}

The equipment was identical to Experiment 1. The tactile pulse used in the Experiment 2 is shown in Fig. 9. The rise time of the pulse was $5 \mathrm{~ms}$ and the fall time $4 \mathrm{~ms}$, displacement of the display module being about $25 \mu \mathrm{m}$, leading a feeling of soft tactile click. When the user pressed a key, this pulse was given after a delay. The delays used were 18, 38, 58, 78, 98, 118, 138 and $158 \mathrm{~ms}$. Given the results of Experiment 1, participants did not wear headphones to block out the audio feedback from the actuators, as this had no statistical effect on the accuracy of the number entry tasks. 


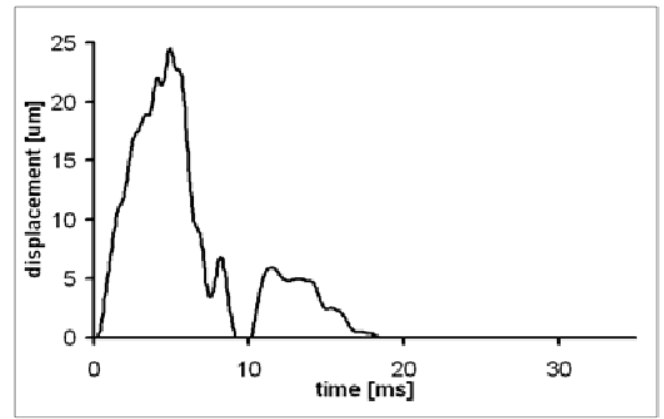

Fig. 9. The tactile feedback pulse used in Experiment 2. The figure shows the displacement of the display module.

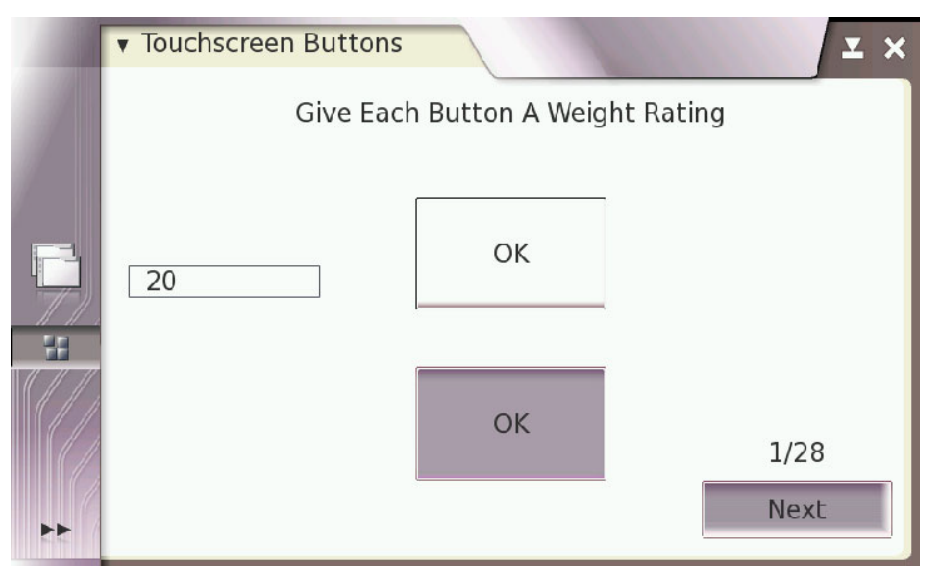

Fig. 10. Screenshot of interface in Experiment 2

\subsection{Methodology}

The aim of this experiment was to investigate whether users perceive buttons with a bigger delay as being heavier. A magnitude estimation method was used where participants assigned an arbitrary number of their own choice to the weight of a baseline stimulus presented. Participants were then presented with a series of further stimuli (buttons plus delay) and asked to assign a weight number reflecting its perceived weight relative to the baseline. Overall there were 28 tasks in the experiment using a random baseline stimulus (chosen from the 7 clicks with different delays). For example, a participant could be presented with the $18 \mathrm{~ms}$ delay stimulus as the baseline and asked to assign a weight value to the $158 \mathrm{~ms}$ delay stimulus in comparison to the baseline (Fig. 10). The experiment took approximately 30 minutes to complete.

Overall, fourteen participants took part in the experiment: 8 male and 6 female, all employees of Nokia Research Center. In general terms, the experiment involved a within subjects design where teach participant completed all magnitude estimation tasks for all seven delay categories. All tasks were counterbalanced. 


\subsection{Hypothesis}

The hypothesis for this experiment was as follows:

1. Longer delays in tactile feedback will result in the perception of heavier buttons.

\subsection{Results}

During the experiment, the experimental software recorded data on the participants' magnitude estimations of each stimulus as shown in Fig. 11. Given that participants could assign any weight rating they wished, all data were normalized prior to analysis.

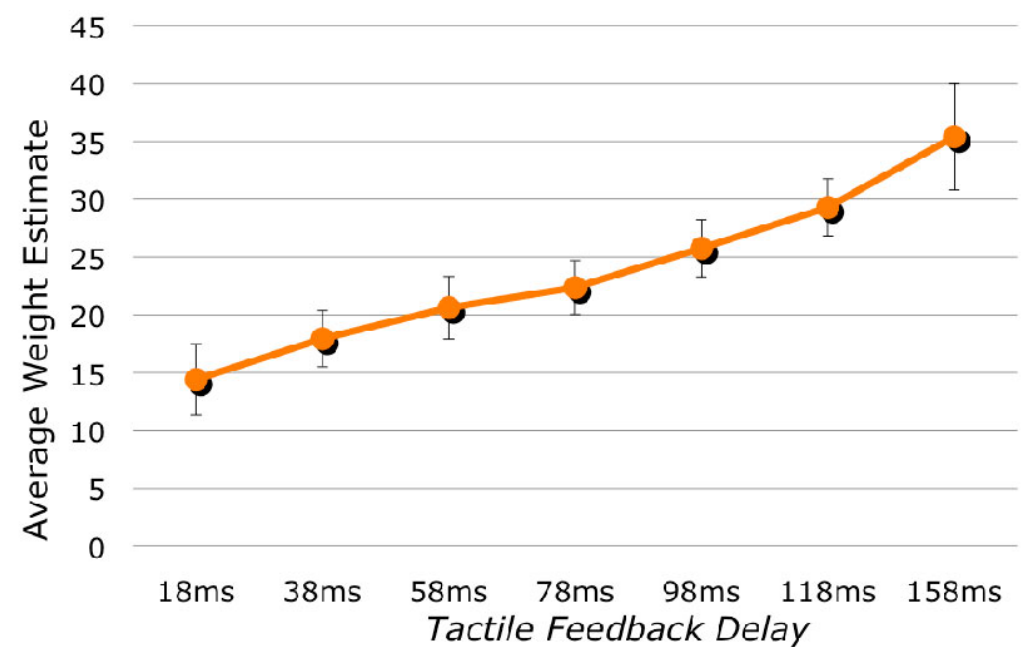

Fig. 11. Average weight estimate for each tactile feedback delay (with standard deviations)

From the average weight estimates for each tactile feedback delay we found a significant correlation of $0.81(\mathrm{r}=0.532, \mathrm{p}=0.05)$ showing a positive relationship between weight and latency. This correlation suggests that higher feedback delays result in the perception of heavier buttons.

Analysis of the weight estimates for each tactile feedback delay using an ANOVA showed a significant difference $(\mathrm{F}=13.69, \mathrm{df}=6, \mathrm{p}<0.05)$. As summarised in Table 1 , Post hoc Tukey tests with Bonferroni corrected significance levels showed that buttons with delays of 78 to $158 \mathrm{~ms}$ are perceived as significantly heavier than those using an $18 \mathrm{~ms}$ delay $(\mathrm{p}=0.05)$. Buttons with delays ranging from 98 to $158 \mathrm{~ms}$ are perceived as significantly heavier than those with a $38 \mathrm{~ms}$ delay $(\mathrm{p}=0.05)$. Buttons with delays of between 118 and $158 \mathrm{~ms}$ are also perceived as significantly heavier than 58 to $78 \mathrm{~ms}$ delays $(\mathrm{p}=0.05)$ and a $158 \mathrm{~ms}$ delay is also perceived as significantly heavier than a $98 \mathrm{~ms}$ delay $(\mathrm{p}=0.05)$. Lastly, there were no significant differences in the perceived weight of touchscreen buttons with delays ranging between 18 and $58 \mathrm{~ms}$ or between 118 and $158 \mathrm{~ms}$. 
Table 1. Summary of tactile latencies (in milliseconds) perceived to create heavier buttons

\begin{tabular}{|c|c|c|c|c|c|c|c|c|}
\hline & 18 & 38 & 58 & 78 & 98 & 118 & 138 & 158 \\
\hline 18 & & & & $*$ & $*$ & $*$ & $*$ & $*$ \\
\hline 38 & & & & & $*$ & $*$ & $*$ & $*$ \\
\hline 58 & & & & & & $*$ & $*$ & $*$ \\
\hline 78 & & & & & & $*$ & $*$ & $*$ \\
\hline 98 & & & & & & & & $*$ \\
\hline 118 & & & & & & & & \\
\hline 138 & & & & & & & & \\
\hline 158 & & & & & & & & \\
\hline
\end{tabular}

These results indicate that it is possible for users to interpret delays as having an effect on the perceived 'weight' of touchscreen buttons. Button presses with tactile feedback delayed by $18 \mathrm{~ms}, 78 \mathrm{~ms}$, and 118 to $158 \mathrm{~ms}$ respectively are perceived as being significantly heavier.

\subsection{Post-study Questionnaire Results}

At the end of the experiment, participants were given the opportunity to try all buttons with all delays again and asked which button they thought was the heaviest and which was the lightest. The results are shown in Fig. 12.

Participants did not rate any of the buttons with short delays as 'heavy'. $158 \mathrm{~ms}$ was voted as the heaviest $57 \%$ of the time and $118 \mathrm{~ms} 43 \%$ of the time. This suggests that the weight difference between 118 and $158 \mathrm{~ms}$ is negligible.

As opposed to the votes for the heaviest button, no long delays were chosen to be the lightest buttons. The shortest delays of 18 and $38 \mathrm{~ms}$ were voted as the lightest buttons (with 1 vote for $78 \mathrm{~ms}$, which is surprising. Further investigation with a larger number of participants is required.)

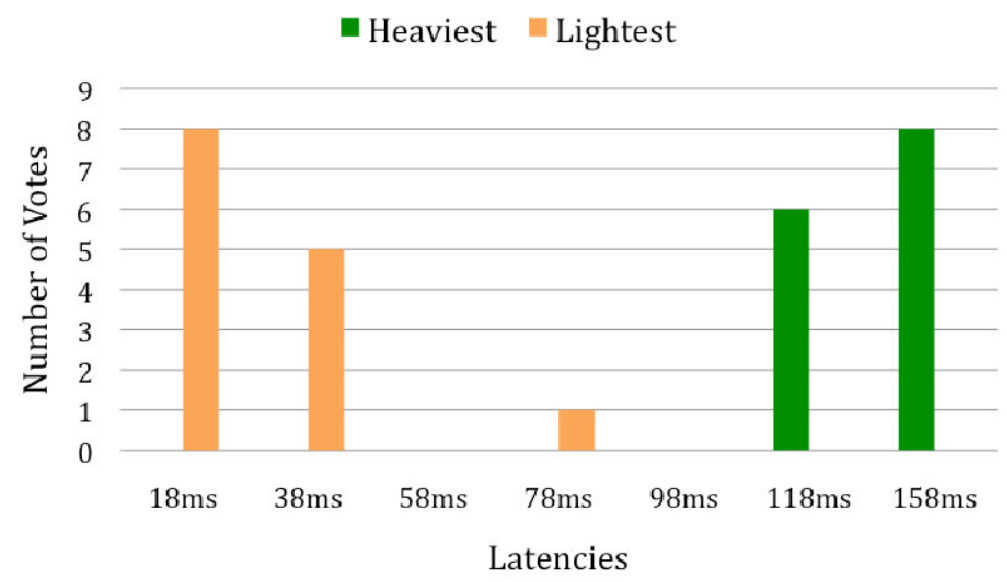

Fig. 12. Total number of votes for the heaviest and lightest button based on delay 
In the post-study questionnaire, participants were also asked to rate levels of pleasantness, comfort, realism and confidence on a scale of 0 to 7 . As can be seen in Fig. 13, all buttons with all delays were rated fairly highly for all measures.

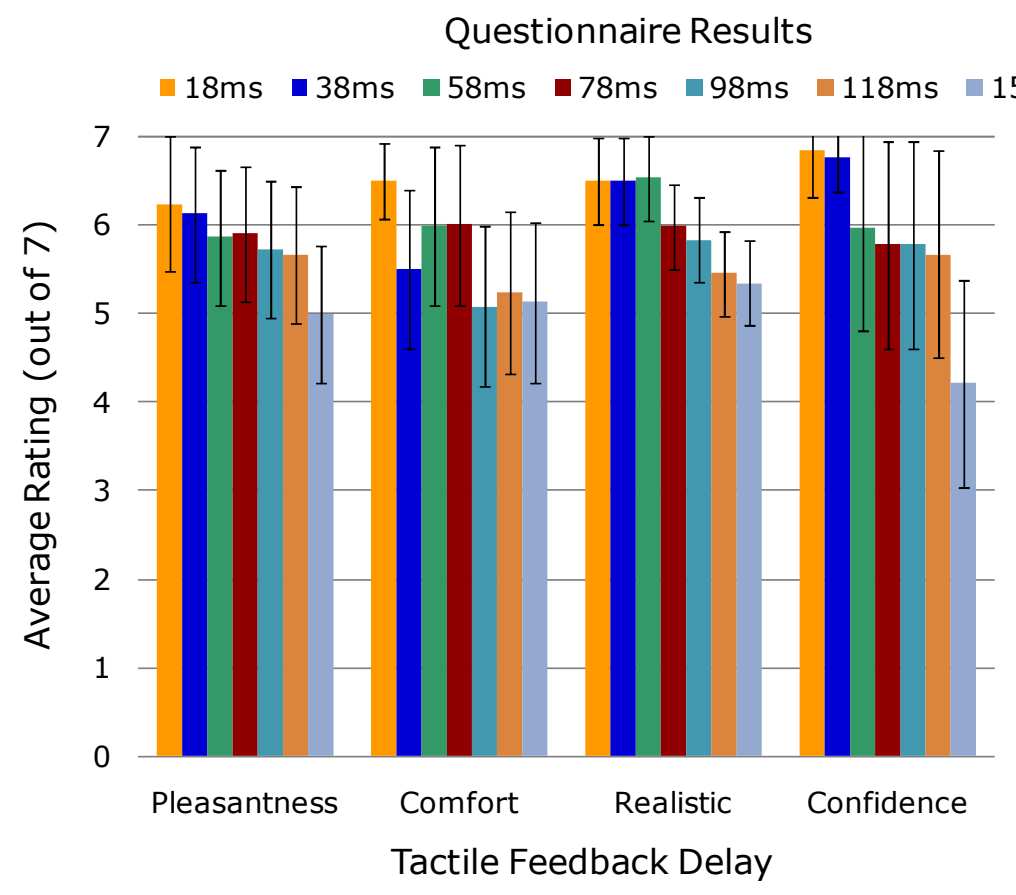

Fig. 13. Average rating for each delay (out of 7) with standard deviations

Analysis of the questionnaire results showed no significant differences except for confidence levels. A Kruskal-Wallis test $(\mathrm{H}=12.87$, $\mathrm{df}=6, \mathrm{p}=0.05)$ followed by post hoc Dunn's Tests $(\mathrm{p}=0.05)$ confirmed that participants experienced significantly lower confidence levels when using buttons with $158 \mathrm{~ms}$ feedback delays compared to those with 18 and $38 \mathrm{~ms}$. Some participants stated: "it (158 ms) seems unresponsive" and "sticky (118 ms and $158 \mathrm{~ms}$ )" which helps to explain such low confidence levels.

\subsection{Discussion}

Overall, when using this range of delays, 3 significantly different weights can be produced using: 18,78 and 118 or $158 \mathrm{~ms}$. One participant mentioned, "only C (158 $\mathrm{ms}$ ) is unpleasant to use". Fortunately, given that feedback delays of $118 \mathrm{~ms}$ are also perceived as significantly heavier than $78 \mathrm{~ms}$ and $18 \mathrm{~ms}$, there is no need to use a delay of $158 \mathrm{~ms}$ when aiming for three distinctive weights.

In the future, tactile latency could be incorporated into touchscreen widgets such as soft keyboards. For example, buttons with important or crucial functions such as 'delete' or 'send' could use a longer feedback delay to create the illusion of weight. 


\section{Overall Discussion and Conclusions}

This paper reported the results of two experiments investigating tactile feedback latencies in touchscreen interaction. Both experiments approached latency from a different perspective. The first experiment took a traditional stance with an aim to establish the levels at which variable tactile feedback latencies become detrimental to performance and perceived pleasantness of touchscreen keypads. The second experiment focused on the potential benefits of latency as a design parameter.

The first experiment produced some interesting findings. Firstly, tactile feedback latencies with small variations between 18 and $72 \mathrm{~ms}$ were found to be acceptable to most users. The results show that the keypad with a feedback delay variation of 18$144 \mathrm{~ms}$ was slowest to use. However, there was no significant increase in the error rate. The majority of participants indicated in the satisfaction survey that high levels of variation (18 to $144 \mathrm{~ms}$ ) were not pleasant or preferred.

In real world systems, it is more likely for users to encounter variable feedback delays than constant delays so it is important for designers to take this into account and ensure that such variations do not exceed $72 \mathrm{~ms}$.

Unexpectedly, the results of the first experiment also indicate that audio feedback does not have an extra effect of the performance and perceived pleasantness of tactile feedback with variable latencies. This is a very positive finding given that most devices produce some intrinsic audio feedback when the tactile actuators vibrate. It is reassuring to know that this extra feedback does not affect performance. However, it must be noted that the additional audio feedback could perhaps cause annoyance to users, especially in discreet situations and environments such as libraries.

Experiment 2 was conducted to establish whether users perceive buttons with a bigger tactile feedback delay as being heavier. The results show that adding tactile feedback latencies to button presses does indeed create the illusion of added button weight. More specifically, to create a set of three different buttons with different perceived weights, the tactile feedback latencies should be set to $18 \mathrm{~ms}, 78 \mathrm{~ms}$ and $118 \mathrm{~ms}+$.

It may seem unusual to encourage the introduction of feedback delays but in this case our research has successfully shown that tactile feedback delays often go unnoticed by users and that, instead, these delays create the illusion of weight. This may explain the high tolerance of users to the tactile feedback latencies in the first experiment discussed in this paper and in our previous work on constant feedback latencies [8].

In order for this new design parameter to be used effectively, it will be necessary to combine it with other tactile parameters to see if there are any negative effects when used in combination. Furthermore, future studies will investigate similar touchscreen widget weight illusions with other combinations of modalities such as audio and visual. The thresholds of this parameter will also be investigated to establish the maximum and minimum delays that can be introduced to create the illusion of weight without disturbing performance. Given the relatively low number of design parameters in the tactile modality, compared to audio for example, the addition of a weight/latency parameter can greatly increase the amount of distinguishable tactile feedback that can be created. This new parameter may be used to enhance many different types of interface widgets and also to represent the weight of 3D objects. 


\section{References}

1. Wright, M., Cassidy, R.J., Zbyszynski, M.F.: Audio and Gesture Latency Measurements on Linux and OSX. In: International Computer Music Conference, Miami, FL, USA, pp. 423-429 (2004)

2. He, D., Liu, F., Pape, D., Dawe, G., Sandin, D.: Video-Based Measurement of System Latency. In: IPT 2000, International Immersive Projection Technology Workshop, Ames IA, USA (2000)

3. Mine, M.R.: Characterization of End-to-End Delays in Head-Mounted Display Systems. University of North Carolina (1993)

4. Zampini, M., Brown, T., Shore, D.I., Maravita, A., Roder, B., Spence, C.: Audiotactile temporal order judgements. Acta Psychologica 118, 277-291 (2005)

5. Zampini, M., Shore, D.I., Spence, C.: Audiovisual temporal order judgements. Experimental Brain Research 152, 198-210 (2003)

6. Lee, J.-H., Spence, C.: Spatiotemporal Visuotactile Interaction. In: Ferre, M. (ed.) EuroHaptics 2008. LNCS, vol. 5024, pp. 826-831. Springer, Heidelberg (2008)

7. Kaaresoja, T., Brewster, S.: Feedback is...Late: Measuring Multimodal Delays in Mobile Device Touchscreen Interaction. In: ICMI 2010. ACM, Beijing (2010)

8. Kaaresoja, T., Anttila, E., Hoggan, E.: The Effect of Tactile Feedback Latency in Touchscreen Interaction. In: World Haptics 2011. IEEE, Istanbul (2011)

9. Poupyrev, I., Maruyama, S., Rekimoto, J.: Ambient Touch: Designing Tactile Interfaces for Handheld Devices. In: UIST 2002, pp. 51-60. ACM, Paris (2002)

10. Nashel, A., Razzaque, S.: Tactile Virtual Buttons for Mobile Devices. In: CHI 2003, pp. 854-855. ACM Press, Ft. Lauderdale (2003)

11. Kaaresoja, T., Brown, L.M., Linjama, J.: Snap-Crackle-Pop: Tactile Feedback for Mobile Touch Screens. In: Eurohaptics, Paris, France, pp. 565-566 (2006)

12. Hoggan, E., Brewster, S.A., Johnston, J.: Investigating the Effectiveness of Tactile Feedback for Mobile Touchscreens. In: CHI 2008, Florence, Italy (2008)

13. Brown, L.M., Brewster, S.A., Purchase, H.C.: Multidimensional Tactons for Non-Visual Infomation Display in Mobile Devices. In: MobileHCI 2006. ACM, Espoo (2006)

14. Adelstein, B.D., Begault, D.R., Anderson, M.R., Wenzel, E.M.: Sensitivity to haptic-audio asynchrony. In: 5th International Conference on Multimodal Interfaces, pp. 73-76. ACM, Vancouver (2003)

15. Mäki-Patola, T., Hämäläinen, P.: Latency Tolerance for Gesture Controlled Continuous Sound Instrument Without Tactile Feedback. In: International Computer Music Conference (ICMC), Miami, USA (2004)

16. MacKenzie, S., Ware, C.: Lag as a Determinant of Human Performance In Interactive Systems. In: CHI 1993. ACM, Amsterdam (1993)

17. Laitinen, P., Mäenpää, J.: Enabling mobile haptic design: Piezoelectric actuator technology properties in hand held devices. In: HAVE 2006 (Haptic Audio Visual Environments), Ottawa, Canada (2006)

18. Hoggan, E., Brewster, S.: New Parameters for Tacton Design. In: CHI 2007. ACM, San Jose (2007)

19. Ternes, D., MacLean, K.E.: Designing Large Sets of Haptic Icons with Rhythm. In: Ferre, M. (ed.) EuroHaptics 2008. LNCS, vol. 5024, pp. 199-208. Springer, Heidelberg (2008) 
20. Murray, D.J., Ellis, R.R., Bandomir, C.A., Ross, H.E.: Charpentier on The Size-Weight Illusion. Perception and Psychophysics 61(8), 1681-1685 (1891) (1999)

21. Ross, H.E., Gregory, R.L.: Weight Illusions and Weight Discrimination - A Revised Hypothesis. Q. J. Exp. Psychol. 22(2), 318-328 (1970)

22. van Mensvoort, I.M.K.: What you see is what you feel: exploiting the dominance of the visual over the haptic domain to simulate force-feedback with cursor displacements. In: DIS 2002, pp. 345-348. ACM, London (2002)

23. Ellis, R.R., Lederman, S.J.: The Role of Haptic Versus Visual Cues in The Size-Weight Illusion. Perception and Psychophysics 53(3), 315-324 (1993)

24. Amazeen, E.L.: Perceptual Independence of Size and Weight by Dynamic Touch. Journal of Experimental Psychology 26(3), 1133-1147 (1999) 